\title{
On the dynamic modelling and simulation of rigid- flexible manipulator robot using several inputs
}

\author{
Sameh Zribi \\ Research Laboratory LARA in Automatic Control, National \\ Engineering School of Tunis (ENIT), University of Tunis El \\ Manar, BP 37, Le Belvédère, 1002 Tunis, Tunisia.
}

Sameh.Zribi@enit.rnu.tn

Jilani Knani

Research Laboratory LARA in Automatic Control, National Engineering School of Tunis (ENIT), University of Tunis El Manar, BP 37, Le Belvédère, 1002 Tunis, Tunisia.

Jilani.Knani@enit.rnu.tn

\begin{abstract}
The purpose of this paper is to develop a dynamic model of a rigid-flexible manipulator robot with a load on its endpoint using Euler-Lagrange formulation. In order to test the performance of the studied system, several mathematical functions are used as motion profile. It choice is very important because it affects the robot's performance. Different factors intervene in this choice. However, the most important is the torque's continuity and the movement's smoothness. Numerical simulations show the robustness of the dynamic model of the studied system for several motions profiles.
\end{abstract}

Keywords-Flexible arm; manipulator; Rigid-flexible robot; dynamic modelling; trajectory generation; motion profile.

\section{Introduction}

The importance of robot manipulators lies in the variety of its structures on the one hand [1], [2] and on the other hand in the variety of its fields of application in the modern industry [3], [4]. In recent decades, the use of flexible bodies in the manipulators robots structure has triggered a great interest in the control domain [5]. There has been an important progress in planning [6], [7], design [8] and control [9].

In rigid-flexible manipulator robots control, especially in high speeds, some difficulties may arise related to the dynamics' complexity of this system such as highly nonlinearity, external disturbance and time-varying and coupled dynamic behaviour [10], [11]. By comparing the method of the assumed modes [12] with that of the finite element, the main advantage of the finite element approach is based on approximating the Euler-Bernoulli links of complex geometrical shapes and of the closed-loop manipulators with fewer computations. This makes this approach more suited for the implementations of a dynamic model-based real-time controller [13]. To deal with these problems, a methodology to obtain the nonlinear dynamical

\author{
Hatem Tlijani \\ Research Laboratory LARA in Automatic Control, National \\ Engineering School of Tunis (ENIT), University of Tunis El \\ Manar, BP 37, Le Belvédère, 1002 Tunis, Tunisia. \\ hatemtlijaniissat@gmail.com \\ Vicenç Puig \\ Department of Automatic Control (ESAII) \\ University Polytechnic of Catalonia, Despatx 525 (Campus \\ Sud) Pau Gargallo, 5, 08028 - Barcelona. \\ vicenc.puig@upc.edu
}

model of the considered system is necessary. By considering the Euler-Lagrangian approach and the finite element method [14], differential equations represent the dynamic model of the rigid-flexible manipulator robot. The work presented in this paper focuses on simulating the obtained dynamic model of our system by applying several motions profile in order to evaluate the system's performances.

This paper is planned as follows: In Section II, the dynamic model of rigid-flexible manipulator robot, using the EulerLagrange method, is detailed. Section III deals with the generation of several motion profiles more used in scientific literature. Simulation results of these motions profiles applied to our rigid-flexible manipulator robot are presented in Section IV. Finally, some concluding remarks are provided in the Section V.

\section{Proposed approach for dynamic modelling of rigid- flexible manipulator robot}

\section{A. Mechanism's description and assumptions}

In this section, a description of a rigid-flexible manipulator robot considered as a case study is given. This system is composed of five rigid bodies and the sixth one is assumed as flexible body (end-effector) in which a mass load denoted by $M$ is fixed in its extremity. The considered robot is constituted by an articulated chain with series architectures. These bodies are manipulated with six joints which are all pivots as shown in Figure 1. These rotary connections are performed by six DC motors, placed directly to the links. This robot is a multivariable, under actuated and non-linear system. Its outputs are the values of its joint angles and its inputs are described by torques provided by the DC motors. 


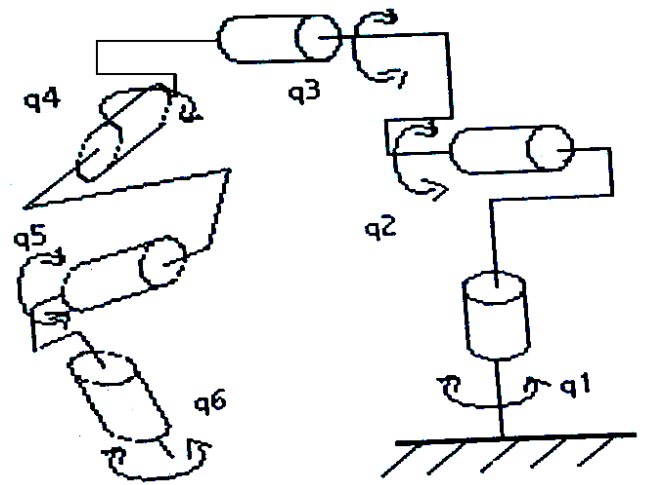

Fig. 1. Connections of rigid-flexible manipulator robot scheme

In order to simplify the modelling calculation, only the last two solids of the mechanism will be modelled. The first solid $C_{1}$ is rigid, whereas, the second $C_{2}$ is assumed as a uniform Euler-Bernoulli beam with a mass load concentrated at its end, which are presented by

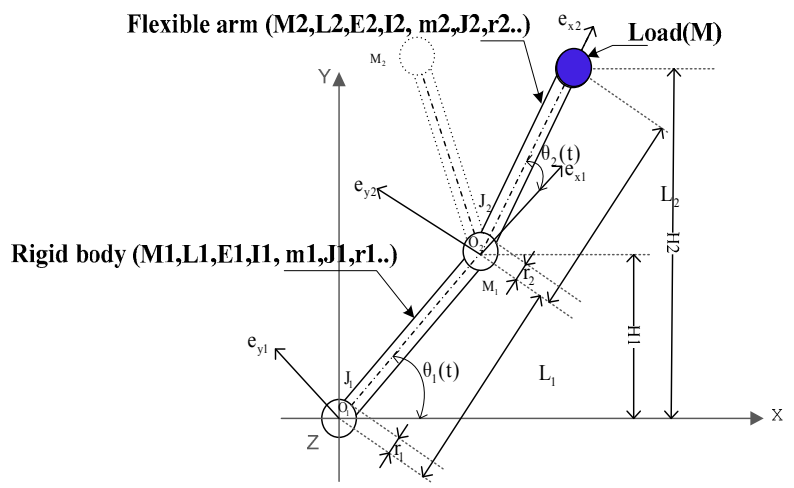

Fig. 2. Two links rigid-flexible manipulator robot with rotary joint

In Figure 2, $M_{i}, L_{i}, E_{i}, I_{i}, l_{i}$ and $m_{i}$ are respectively the mass, the length, the Young's modulus, the area moment of inertia, the length of the $i^{\text {th }}$ element, the mass per length's unit of $i^{\text {th }}$ solid and $r_{i}, J_{i}$ describe respectively the radius and the inertia's moment of the $i^{\text {th }}$ articulation. Moreover, $\theta_{i}(t)$ is the rotation's angle of the solid $i^{\text {th }}$ around the axis $Z$. The following modelling assumptions will be considered: the robot's motion is supposed to be in the vertical plane. For the flexible arm, the shear strain, the effect of the axial force and the rotational inertia are assumed to be negligible. In order to avoid the problems and difficulties arising when the length of this flexible arm is variable, this length is considered to be constant. Besides, his depth is assumed to be smaller than its length which allows the manipulator to vibrate mainly in the horizontal direction.

\section{B. Dynamic modeling}

In this section, the determination of a dynamic model of the considered robot will be detailed. To develop the model, the fundamental principles of dynamics and kinematics, the finite elements and Lagrange's equations will be used. In the remainder of this paper, a number in brackets in the power of such a function has the usual meaning of function's time derivation.

The Euler-Lagrange formulation is described as follows [14]

$$
\frac{d}{d t}\left(\frac{\partial L}{\partial q^{(1)}}\right)-\frac{\partial L}{\partial q_{k}}=Q_{n}
$$

with

$$
L=E_{c T o t}-E_{p T o t}
$$

where $k$ is body index $(k=1,2), E_{\text {cTot }}$ represents the total kinetic energy of the robot, $E_{p T o t}$ states the total potential energy of the robot, $Q_{n}$ presents the vector of generalized forces, $q_{i}$ and $q^{(1)}{ }_{i}$ are, respectively, the vector of joints angles and its velocities. The calculation of kinetic and potential energies is performed from the elemental energies of each body.

The total kinetic energy $E_{\text {cTot }}$ is evaluated as the sum of the kinetic energy of rigid body and that of flexible body. In general, it is given by

$$
E_{\text {cTot }}=\frac{1}{2} J_{1} \vec{w}_{i} \vec{w}_{i}+\frac{1}{2} \int_{r_{i}}^{l_{i}} m_{i} \vec{V}_{i} \vec{V}_{i} d x_{i}+\left.\frac{1}{2} M_{i} \vec{V}_{i} \vec{V}_{i}\right|_{x_{i}=l_{i}}
$$

where $w_{i}$ is the absolute angular speed of the system and $V_{i}$ is the absolute speed of any point of the $i^{\text {th }}$ body.

For a rigid body, its kinetic energy $T_{1}$ is defined by

$$
T_{l}=\frac{1}{2} J_{l}\left(\theta^{(1)}{ }_{1}\right)^{2}+\frac{1}{2} \int_{r_{1}}^{l_{l}} m_{l}\left(\theta^{(1)}{ }_{1}\right)^{2} d x_{1}+\frac{1}{2} M_{l}\left(l_{l} \theta^{(1)}{ }_{1}\right)^{2}
$$

and it can be obtained as follows

$$
T_{1}=\frac{1}{6}\left[\left(l_{1}^{3}-r_{1}^{3}\right) m_{1}+3 J_{1}+3 M_{1} I_{1}^{2}\right]\left(\theta_{1}^{(1)}\right)^{2}
$$

For flexible body which is described by uniform EulerBernoulli beam [15], it is assumed subdivided into two elements. So, its kinetic energy is described by

$$
T_{2}=\frac{1}{2} J_{2}\left(\theta_{1}^{(1)}{ }_{1}+\theta_{2}^{(1)}{ }_{2}\right)^{2}+\frac{1}{2} \int_{r_{2}}^{l 2} m_{2} V_{2}^{T} V_{2} d x_{2}+\left.\frac{1}{2} M_{2} V_{2}^{T} V_{2}\right|_{x_{2}=l_{2}}
$$

In fact, it presents the sum of kinetic energy $T_{2 r}$ of rigid part of flexible arm and that $T_{2 f}$ of flexible part of this arm. The energy $T_{2 r}$ can be obtained as follows

$$
\begin{aligned}
T_{2 r} & =\frac{1}{6}\left[\left(r_{2}^{2}-l_{2}^{2}\right) m_{2}+3 J_{2}+3 M_{2} l_{2}^{2}\right]\left(\theta_{1}^{(1)}+\theta_{2}^{(1)}\right)^{2} \\
& +\frac{1}{2}\left[m_{2} l_{2}^{2}\left(l_{2}-r_{2}\right)+M_{2} l_{1}^{2}\right]\left(\theta_{1}^{(1)}\right)^{2} \\
& +\frac{1}{2}\left[m_{2} l_{1}\left(l_{2}^{2}-r_{2}^{2}\right)+2 M_{2} l_{1} l_{1}\right]\left(\theta_{1}^{(1)}+\theta_{2}^{(1)}\right) \theta^{(1)} C_{2}
\end{aligned}
$$


In all expressions, we denote by $S_{i}$ the trigonometric function $\sin \left(\theta_{i}\right)$ and by $C_{i}$ the trigonometric function $\cos \left(\theta_{i}\right)$. According to generalized coordinates, the energy $T_{2 f}$ is expressed as follows

$$
\begin{aligned}
T_{2 f} & =\frac{1}{2} \eta^{T} M_{f f} \eta\left(\theta_{1}^{(1)}+\theta_{2}^{(1)}\right)^{2}+\frac{1}{2} \eta^{(1) T} M_{f f} \eta^{(1)} \\
& +\left(\theta^{(1)}{ }_{1}+\theta^{(1)}{ }_{2}\right)\left[M_{f \theta_{2}} \eta^{(1)}-M_{f \theta_{l}} \eta l_{1} \theta^{(1)}{ }_{1} S_{2}\right] \\
& +M_{f \theta_{l}} \eta^{(1)} l_{l} \theta^{(1)}{ }_{l} C_{2}
\end{aligned}
$$

where $\eta$ is the vector of nodal variables of the elementary bodies of the flexible arm, $\eta^{(1)}$ is the vector of its speeds, $M_{f f}$ is the mass matrix of the flexible part deduced from the Hermite spline function, $M_{f \theta_{l}}$ and $M_{f \theta_{2}}$ present respectively the matrix corresponding to the centrifugal effect and that of Coriolis for the flexible part.

For the total potential energy $E_{p T o t}$, it is composed of the strain energy $U_{a}$ produced by the centrifugal forces at a point on the Bernoulli's beam, the strain energy $U_{b}$ due to deformation by deflection, the energy $U_{c}$ produced by the engines and the potential energy $U_{g}$ due to gravity. The energy $U_{a}$ can be deduced from the following equation

$$
-\delta U_{a}=-\int_{r_{2}}^{l_{2}} R\left(x_{2}\right)\left(\frac{\partial y_{2}}{\partial x_{2}}\right) \delta\left(\frac{\partial y_{2}}{\partial x_{2}}\right) d x_{2}
$$

where $R\left(x_{2}\right)$ is the axial load of a point of the Bernoulli beam located at $x_{2}$ and $y_{2}$ is the elementary displacement which can be given by

$$
y(x, t)=\sum_{i=1}^{4} \phi_{i} U_{i}(t)
$$

where $\phi_{i}$ is the Hermite spline functions and $U_{i}(t)$ is the movement (translation or rotation) of each fictitious joint of the flexible arm [16]. In order to simplify its dynamic modelling, the flexible arm is assumed to be divided into four elements. The energy $U_{b}$ is expressed by

$$
U_{b}=\frac{1}{2} \int_{r_{2}}^{l_{2}} E_{2} I_{2}\left(\frac{\partial y_{2}}{\partial x_{2}{ }^{2}}\right)^{2} d x_{2}
$$

Concerning the energy $U_{c}$, it can be described by

$$
U_{c}=-\sum_{i=1}^{2} u_{i} \theta_{i}
$$

where $u_{i}$ is the $i^{t h}$ input torque provided by the $i^{\text {th }}$ actuator. Thus, the energy $U_{g}$, it is given by

$$
\begin{aligned}
U_{g} & =\sum_{i=1}^{2}\left[\int_{r_{2}}^{l_{2}} m_{2} y_{2} d x_{2}+\left.M_{2} y_{2}\right|_{x_{2}=l_{2}}\right] g \cos \left(\theta_{1}+\theta_{2}\right) \\
& +\sum_{i=1}^{2}\left[m_{i} s_{i} h_{i}\left(\theta_{1}, \theta_{2}\right)+M_{i} H_{i}\left(\theta_{1}, \theta_{2}\right) \vec{g}\right.
\end{aligned}
$$

where $H_{i}$ is the height of the $i^{\text {th }}$ body and $h_{i}$ is that of the gravity center of the $i^{\text {th }}$ body, both with respect to the base. After calculating the kinetic and potential energies, the Lagrangian is deduced. Its derivatives according to the vector of joint variables with respect to time are obtained. In terms of generalized coordinates, the dynamic model of the system can be deduced as follows

$$
\begin{aligned}
& {\left[M+M_{n}(q)+M_{a}(q)\right] q^{(2)}+D q^{(1)}} \\
& +\left[K+K_{a}\left(q, q^{(1)}\right)\right] q+N\left(q, q^{(1)}\right)=B u
\end{aligned}
$$

where the sum of $M$ and $M_{a}(q)$ is the matrix of inertia due to rotation induced by the axial load where the first matrix is linear whereas the second is nonlinear, $M_{n}(q)$ is the matrix of inertia due to the additional rotation produced by the axial load which is nonlinear, $D$ is the damping matrix which is linear, $K$ is the generalized geometric stiffness matrix which is nonlinear, $K_{a}\left(q, q^{(1)}\right)$ is the geometric stiffness matrix due to the rotation induced by the axial load which is linear, $N\left(q, q^{(1)}\right)$ is the sum of the vector of gravity's torque and the vector of inertial forces, $B$ is the input matrix and $u$ is the input torques produced by the actuators.

\section{Dynamic model linearization}

The dynamic model obtained of the robot is nonlinear. This nonlinearity appears because of the trigonometric functions at a few elements of the characteristic matrix. The linearization of our system is developed around any point defined by the joint angles $\theta_{0}=\left[\begin{array}{ll}\theta_{10} & \theta_{20}\end{array}\right]$ and with the static deflection $\eta_{0}$. In fact, the vector of joints angles $q$ is decomposed as follows

$$
q=\left[\begin{array}{l}
\theta \\
\eta
\end{array}\right]=\left[\begin{array}{l}
\theta_{0} \\
\eta_{0}
\end{array}\right]+\left[\begin{array}{l}
\theta_{p} \\
\eta_{p}
\end{array}\right]=q_{0}+q_{p}
$$

where $q_{0}$ is the vector of the joint angles around point of linearization and $q_{p}$ is the vector of the small variations of the angles and deflection. As the vector's components of $q_{p}$ are small, the following approximation will be taken into consideration

$$
\theta_{p} \ll 1
$$

By applying the previous approximation to the trigonometric functions, we obtain 


$$
\left\{\begin{array}{l}
\sin \theta_{i}=\sin \left(\theta_{i 0}+\theta_{i p}\right) \simeq \sin \theta_{i 0}+\theta_{i p} \cos \theta_{i 0} \\
\cos \theta_{i}=\cos \left(\theta_{i 0}+\theta_{i p}\right) \simeq \cos \theta_{i 0}-\theta_{i p} \sin \theta_{i 0}
\end{array}\right.
$$

The linearization strategy consists in approximating the nonlinear equations and obtained a linear state space model.

\section{Trajectory generation's description}

The considered system, containing a flexible part, produces vibrations which can hinder the system's performances. Moreover, the motion profile applied to this system causes a vibration phenomenon by providing discontinuous torque as input. Therefore, the specific trajectory to choose must take into account the flexibility of the system .on the one hand and its physical parameters on the other [17]. In the following, several motions profiles will be applied to our system in order to evaluate its performances.

\section{A. Bang-bang motions profiles}

In the literature, the first trajectory planning techniques are based on algorithms with minimal time. The mathematical principle known as bang-bang profiles and formulated by Hermes [17] are the base of these algorithms. This principle consists in guaranteeing the saturation of the system control variable, or one of its derivatives, by switching a number of times the minimum level to the maximum allowable level. This saturation allows optimizing the time because the movement of a system from an initial position to a final position in minimum time using at any moment the maximum power available and saturating the actuators. Figure 3 describes the Hermes principle

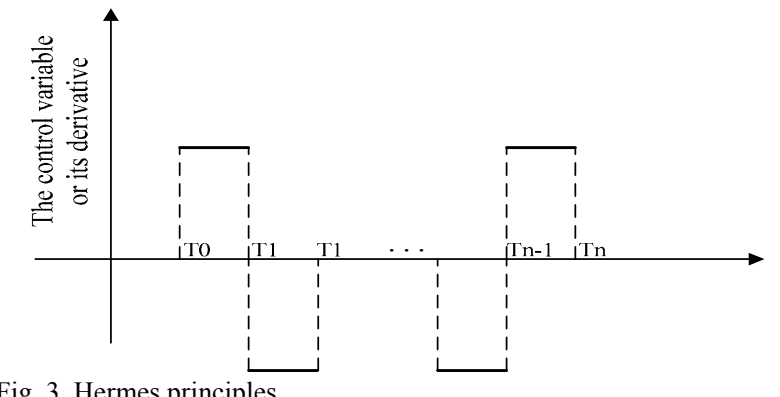

Using the bang-bang motion profile, the minimum time is provided by saturating acceleration. The trapezoidal profile allows to generate a continuous motion speed in which a minimum time is ensured by saturating both the acceleration and speed. Its joint position may be expressed as follows

$$
\begin{cases}q(t)=a_{i} t^{2}+b_{i} & 0 \leq t \leq \tau \\ q(t)=c_{i}\left(t-\frac{\tau}{2}\right)+d_{i} & \tau \leq t \leq T-\tau \\ q(t)=e_{i}(t-T)^{2}+f_{i} & T-\tau \leq t \leq T\end{cases}
$$

where $q(t)$ is the joint position according to time, $\tau$ is the duration of the acceleration phase, $T$ is the duration of movement and $a_{i}, b_{i}, c_{i}, d_{i}, e_{i}$ and $f_{i}$ are constants depending on the initial and the final positions. So, the acceleration of this motion profile can be expressed as follows

$$
\begin{cases}q^{(2)}(t)=2 a_{i} & 0 \leq t \leq \tau \\ q^{(2)}(t)=0 & \tau \leq t \leq T-\tau \\ q^{(2)}(t)=2 e_{i} & T-\tau \leq t \leq T\end{cases}
$$

with a motion time of 6 seconds, from an initial position at $0 \mathrm{rad}$ to a final position of $25 \mathrm{rad}$. Using this profile, we obtain the joint acceleration for this profile presented in Figure 4.

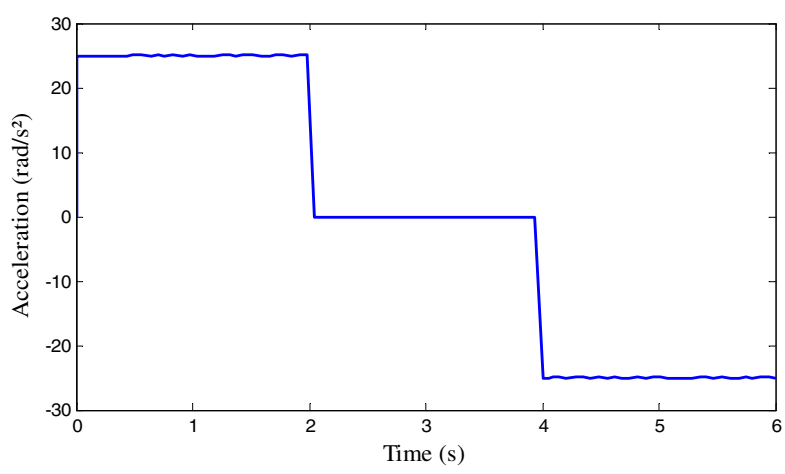

Fig.4. Joint acceleration for trapezoidal profile

For $t=\tau$ and $t=T-\tau$, we notice that the problem of acceleration discontinuity still exists. In order to remedy this discontinuity, another input called polynomial motion profiles will be presented.

\section{B. Polynomial motions profiles}

Alternatively to the polynomial motion profile that presents finite jerk over a period of time, the trapezoidal motion profile presents large spikes in jerk. This quality contributes to lower vibration for the polynomial profiles [18]. Finite jerk implies a continuous acceleration. The three most common methods of this motion profile are described by the third-polynomial interpolation, the linear interpolation and the fifth-polynomial interpolation. In this work, we are interested to consider the fifth-polynomial motion profile described by

$$
q(t)=\left[\frac{q_{f 1}-q_{i 1}}{T^{3}}\right]\left[\frac{6 t^{5}}{T^{2}}-\frac{15 t^{4}}{T}+10 t^{3}\right]+q_{i 1}
$$

where $q_{i 1}$ is the initial position and $q_{f 1}$ is the final position. By deriving the previous equation according to time, we obtain the acceleration relative to this motion profile as follows

$$
q^{(2)}(t)=\left[\frac{q_{f 1}-q_{i 1}}{T^{3}}\right]\left[\frac{120 t^{3}}{T^{2}}-\frac{180 t^{2}}{T}+60 t\right]
$$


Because of space limitation, here we present just the joint acceleration for the fifth-polynomial profile as shown in Figure 5.

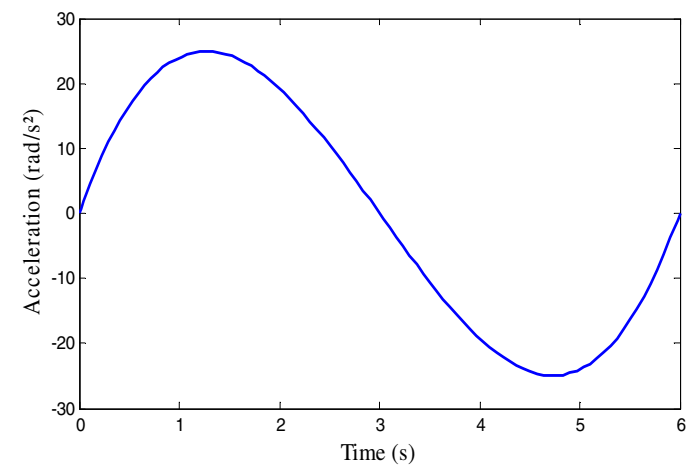

Fig.5. Joint acceleration for fifth-polynomial profile

\section{Hyperbolic motion pofile}

The hyperbolic motion profile is the best-known example of accelerated motion. It is characterized by constant proper acceleration [18] which can be defined by

$$
q^{(2)}(t)=\frac{\left(q_{f 1}-q_{i 1}\right)\left(30 t^{2}\right)}{2 \psi\left(t_{f}-\psi\right)}+q_{i 1}
$$

with

$$
\psi=\frac{2\left(R-q_{f 1}\right)-(1+R) q_{i 1}}{k_{v}}
$$

where $R$ is the instantaneous speed, $k_{v}$ is the maximum speed and $t_{f}$ is the final time of simulation. The joint acceleration of the hyperbolic motion profile is represented as Figure 6.

\section{Iv. Numerical Simulations}

\section{A. Control design}

After determining the dynamic model of rigid-flexible manipulator robot described by (14), we apply the torque to each joint for several motions profiles. The numerical values of parameters of rigid-flexible manipulator robot are presented in Table 1..

Table 1 Dynamic Parameters

\begin{tabular}{ccc}
\hline Parameter & Value & Unit \\
\hline$E_{1}$ & 71 & $\mathrm{GPa}$ \\
$E_{2}$ & 196 & $\mathrm{GPa}$ \\
$I_{1}=I_{2}$ & $1.6710^{-12}$ & $\mathrm{~m}^{4}$ \\
$m_{1}=m_{2}$ & 0.831 & $\mathrm{Kg} / \mathrm{m}$ \\
$r_{1}=r_{2}$ & 0.05 & $\mathrm{~m}$ \\
$J_{1}=J_{2}$ & 0.05 & $\mathrm{Kg} . \mathrm{m}^{2} / \mathrm{rad}$ \\
$L_{1}=L_{2}$ & 0.375 & $\mathrm{~m}$ \\
$M_{1}$ & 0.05 & $\mathrm{Kg}$ \\
$M_{2}$ & $(0.05)^{2}$ & $\mathrm{Kg}$ \\
$M$ & 2.5 & $\mathrm{Kg}$ \\
\hline
\end{tabular}

In order to simulate the considered robot, in which we apply some motions profiles, we resort to a PID controller whose parameters are fixed to the numerical values presented in Table 2. In the remainder of this work and in order to test the robustness of the rigid-flexible manipulator robot using motion profile, we apply several inputs. For example, we chose trapezoidal profile, then fifth polynomial profile and ending with hyperbolic profile.

Table 2 PID controller parameters

\begin{tabular}{ccc}
\hline Parameter & Value & Unit \\
\hline$K_{P 1}$ & 500 & - \\
$K_{I 1}$ & 1 & - \\
$K_{D I}$ & $10^{-3}$ & - \\
$N_{1}$ (Filter coefficient) & 50 & - \\
\hline
\end{tabular}

B. Simulation results

The following figures summarize the simulation results in which the model of rigid-flexible manipulator robot is tested using the PID controller for each joint.

Case 1: For trapezoidal motion profile

With a trapezoidal motion profile, the simulation of the torque applied to the second joint is presented in Figure 7.

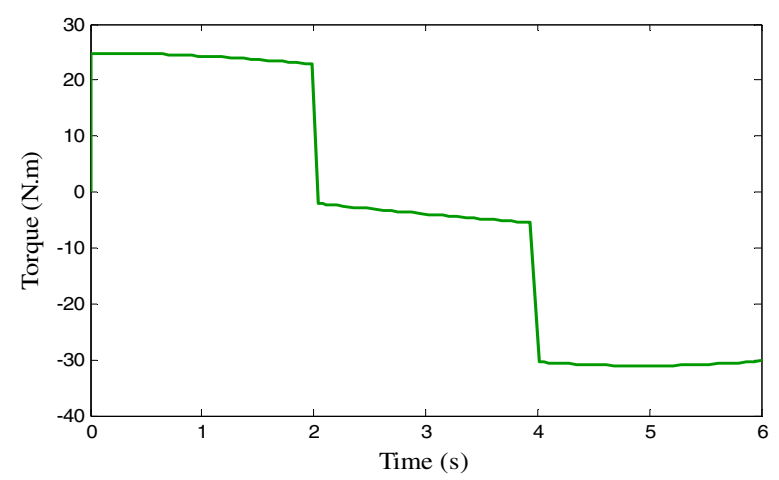

Fig.7. Torque applied to the second joint for trapezoidal profile

It is also important to note that, besides to flexibility problem, the torque discontinuity problem of the trapezoidal motion profile degrades the performances of the system as it will be clearly presented in Fig. 10.

Case 2: For fifth-polynomial motion profile

The fifth-polynomial profile is a sinusoidal shape with values included between $25 \mathrm{~N} . \mathrm{m}$ and $-25 \mathrm{~N} . \mathrm{m}$ for the second joint is shown in Figure 8.

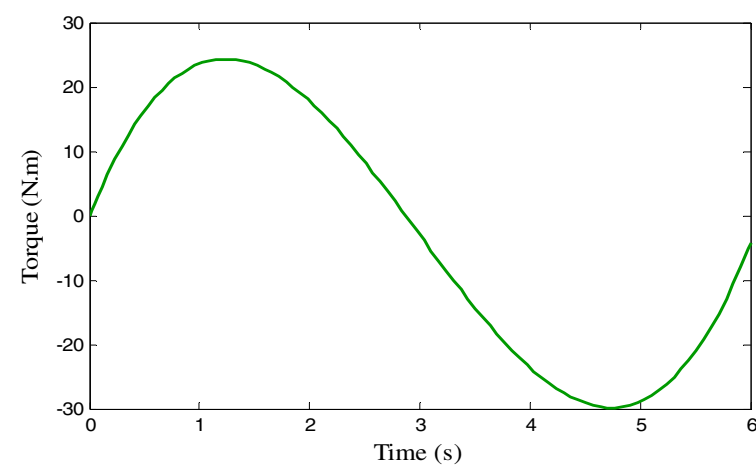

Fig.8. Torque applied to the second joint for fifth-polynomial profile 
It is clear that this torque variation is smoother than that of the trapezoidal profile. The fifth-polynomial profile provides a smooth movement called a minimum-jerk movement. It is similar to that of movement of human.

Case 3: For hyperbolic motion profile

For hyperbolic motion profile, the variation of torque applied to the second joint according to the time is presented in Figure 9.

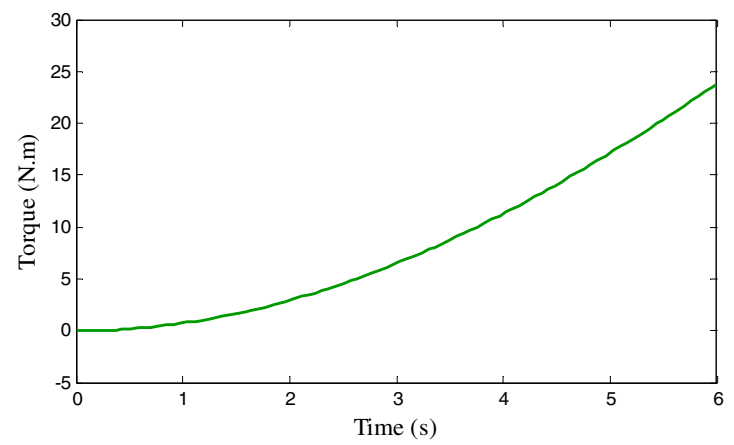

Fig.9. Torque applied to the second joint for hyperbolic profile

From this figure, we can observe that the torque continuity for this joint is ensured via this profile more than that in the case of the previous profiles. To properly compare the three previous cases, Figure 10 shows the tracking errors for each motion profile. We can observe that the tracking error for hyperbolic profile is characterized by slow-moving with low-amplitude compared to that obtained for the two others profiles.

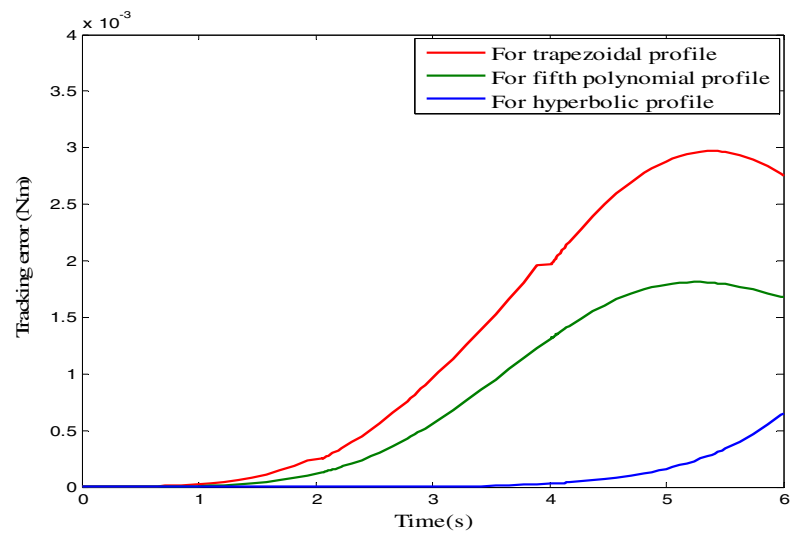

Fig. 4. Tracking errors

\section{v. Conclusion}

In this work, we were interested to simulate the rigidflexible manipulator robot with a load on its endpoint. For this purpose, this system is dynamically modelled using the Euler-Lagrange approach and the finite element method. After obtaining the dynamic model linearization, several motions profiles are applied to the considered system. The contribution of this paper relies on evaluating the system behaviour by adding to the internal perturbation created by the flexible structure of its second arm an external vibration provided by the discontinuous torque applied to the system in a first test and then adding a smooth torque as a motion profile. An improvement in the system performance is obtained through the choice of motion profile characterized by the torque continuity and the movement smoothness.

\section{References}

[1] A. Shawky, D. Zydek, Y. Z. Elhalwagy and A. Ordys, "Modeling and nonlinear control of a flexible-link manipulator", Applied Mathematical Modelling, Vol. 37, pp. 9591-9602, 2013.

[2] C. L. Chen, T. K. Wang, C. J. Hu, and P. C. Lin, "Model-based dynamic gait in a quadruped robot with waist actuation", International Conference on Intelligent Robots and Systems, pp. 2056-2061, Korea, 2016.

[3] X. Yang and S. S. Ge, "Dynamics and tracking control for a freeflying space robot with rigid-flexible links", International Conference on Information and Automation, pp. 394-399, China, 2014.

[4] T. Camarano, J. Beck, B. Li, W. Wu, L. Chow, T. Wu, D. Drumm, M. Lebouitz and D. Napolitano, "Kinematic and dynamic modeling of robot manipulator for golf swing training system”, IEEE International Conference on Industrial Technology, pp. 309-314, Spain, 2005.

[5] D. Ma, Y. Xia, T. Li and K. Chang, "Active disturbance rejection and predictive control strategy for a quadrotor helicopter", IET Control Theory and Applications, Vol. 10, No. 17, pp. 2213-2222, 2016.

[6] B. Tarvirdizadeh and K. Alipour, "Trajectory optimization of twolink rigid flexible manipulators in dynamic object manipulation missions", $3^{\text {rd }}$ International Conference on Robotics and Mechatronics, pp. 493-498, Iran, 2015.

[7] B. Siciliano, L. Sciavicco, L. Villani and G. Oriolo, "Robotics: modelling, planning and control", Advanced Textbooks in Control and Signal Processing, p. 231, 2009.

[8] D. Gurdan, J. Stumpf, M. Achtelik, K. M. Doth, G. Hirzinger and D. Rus, "Energy-efficient autonomous four-rotor flying robot controlled at $1 \mathrm{kHz}$ ", IEEE International Conference on Robotics and Automation, pp. 361-366, Italy, 2007.

[9] K. Khorasani, "Adaptive control of flexible-joint robots", IEEE Transactions On Robotics and Automation, Vol. 8, No. 2, 1992.

[10] C. C. Cheng, S. H. Chien and F.-C. Shih, "design of robust adaptive variable structure tracking controllers with application to rigid robot manipulators", IET Control Theory and Applications, Vol. 4, No. 9, pp. 1655-1664, 2010.

[11] V. Falkenhahn, T. Mahl, A. Hildebrandt, R. Neumann and O. Sawodny, "Dynamic modeling of constant curvature continuum robots using the Euler-Lagrange formalism", International Conference on Intelligent Robots and Systems, pp. 2428-2433, Chicago, 2014.

[12] M. Saad, J.C. Piedboeuf, O. Akhrif and L. Saydy, "Modal analysis of assumed mode models slewing of a flexible beam", International Journal of Modelling, Identification and Control, p325- 337, 2006.

[13] R. J. Theordore and A. Ghosal, "Comparison of the assumed modes and finite element models for flexible multilink manipulators", International Journal of Robotics Research, p. 22, 1995.

[14] H. Roy Pota, "finite-element/Lagrange modeling and control of a flexible robot arm", 11th International Federation of Automatic Control. World Congress, Vol. 9, pp. 239-243, Estonia, 1990.

[15] J. Knani, "Dynamic modelling of flexible robotic mechanisms and adaptive robust control of trajectory computer simulation-Part I", Applied Mathematical Modelling, Vol. 26, pp. 1113-1124, 2002.

[16] M.O. Tokhi and A.K.M.Azad, "Flexible robot manipulators: modeling, simulation and control", IET Control Theory and Applications, 2008.

[17] H. Hermes and J.P Lasalle, "Functional analysis and time optimal control", Academic Press, New York, Vol. 56, 1969.

[18] S. Zribi, M. Mejerbi, H. Tlijani and J. Knani, "Comparison between motions profiles applied to flexible manipulator arm", 3rd International Conference on Automation, Control Engineering and Computer Science, Tunisia, 2016. 